\title{
Due nuovi curculionidi endogei (Coleoptera) (XXXIV. Contributo alla conoscenza della curculionidofauna endogea)
}

di

\author{
G. OSELLA *
}

Con 8 figures

\section{ABSTRACT}

Two new blind endogean weevils (Coleoptera). - Two new blind molytine weevils are described and illustrated. Ruffodytes nitidipennis n. sp. from Greece is characterized by finely punctured elytra, long sculptured legs, and a regularly curved rostrum. Pseudoalaocybites aelleni n. sp. from Curaçao differs from all described species in the finely punctured and shining pronotum and elytra, and very thin and long legs. Lymantes squamirostris (Osella) n. comb. from Venezuela is transferred from Pseudoalaocybites. Keys to species are provided for Ruffodytes and Pseudoalaocybites (Croizatius). I Colleghi Dr. Claude Besuchet e Dr. Berndt Hauser del Museo di Ginevra mi hanno inviato in studio i Curculionidi del suolo raccolti in diverse regioni del Mondo. Due di essi sono l'oggetto della presente nota.

Ringrazio di cuore i Colleghi per l'invio di questo interessante materiale unitamente all'amico R. Pace cui debbo i disegni che corredani il presente lavoro.

Ruffodytes nitidipennis n. sp. (figg. 1-6)

loc. tip.: Iraklia (Cicladi, Grecia)

Materiale esaminato: 1 e etichettata «Isola di Iraklia, 26.IV.1987, leg. B. Hauser»; holotypus Mus. Ginevra.

DiAGNOSI: Specie facilmente riconoscibile per le elitre appena percettibilmente punteggiate, per le zampe gracili ed allungate e per il rostro regolarmente ricurvo dalla base. L'Aquila.

* Università degli Studi dell'Aquila, Dip.to di Scienze Ambientali, Via S. Sisto 20, I-67100 

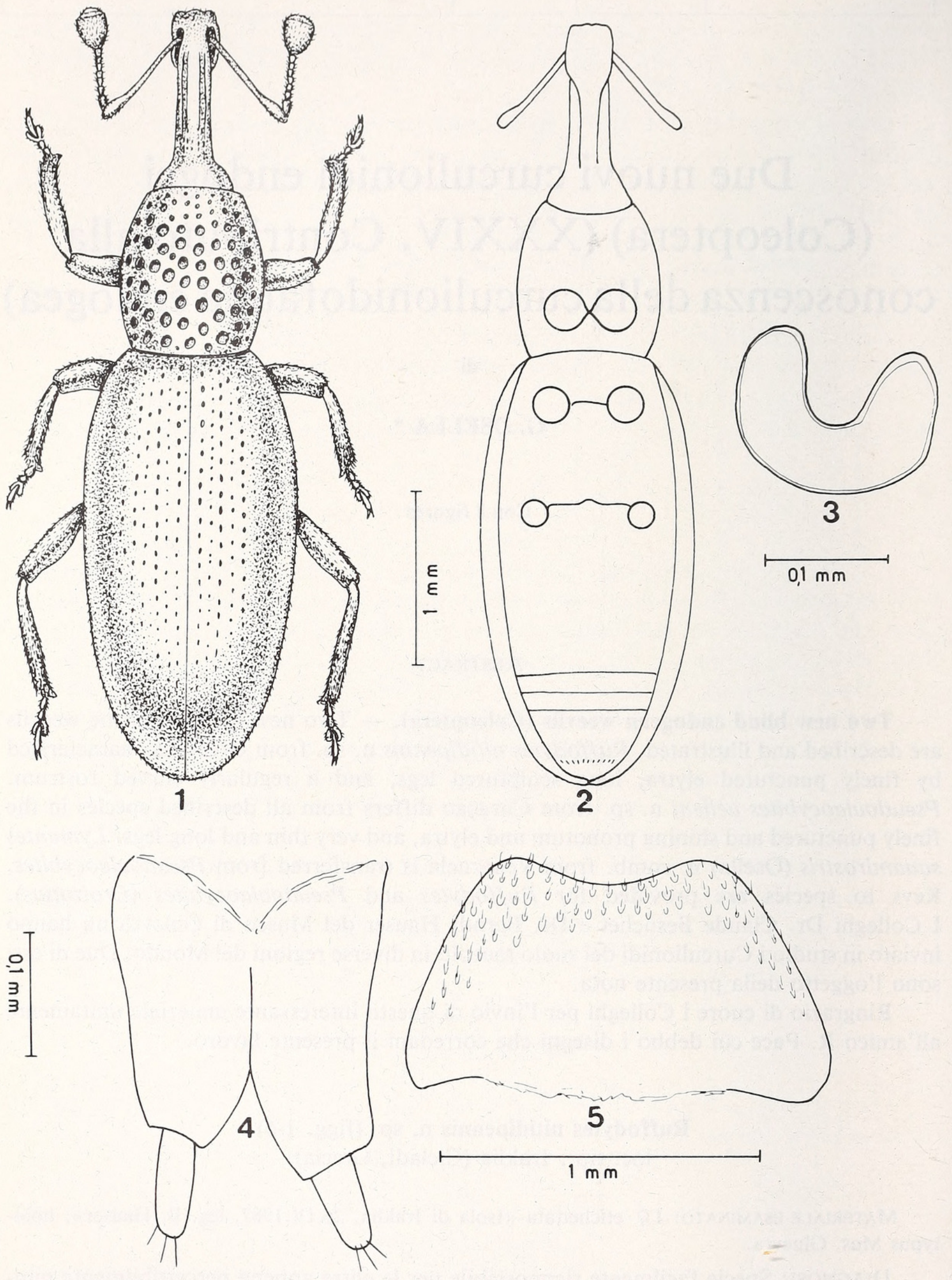

FIGG. 1-5.

Ruffodytes nitidipennis $\mathrm{n}$. sp., Iraklia (Cicladi), holotypus $Q$ : habitus (1), schematico dal ventre (2), spermateca (3), ovopositore (4), ultimo ventrite (5). 
Misure Dell'Holotypus. Lunghezza complessiva del corpo: con il rostro mm 4,46;

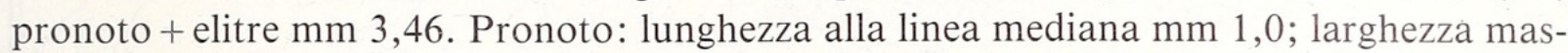

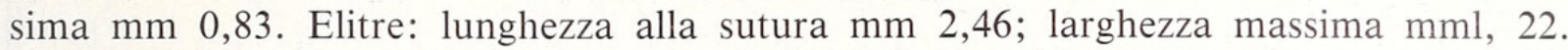
Antenne: scapo mm 0,69; funicolo mm 0,37; clava mm 0,22.

DESCRIZIONE DELL'HOLOTYPUS. Corpo di color rosso chiaro uniforme, punteggiatura delle elitre svanita, più evidente invece quella del pronoto. Pronoto ed elitre ricoperte di setoline cortissime ben visibili solo di profilo. Rostro regolarmente ricurvo con striature longitudinali tra la fronte e le scrobe, spatolato nella parte terminale. Antenne brevi, scapo sottile che, all'indietro, non raggiunge la base del rostro; funicolo compatto, leggermente setoloso, di grossezza uniforme, $1^{\circ}$ articolo cilindrico, all'incirca due volte più lungo che largo, $2^{\circ}$ anch'esso cilindrico, più lungo che largo, restanti trasversi; clava molto grossa con $1^{\circ}$ articolo più lungo che largo, restanti trasversi, setolosi. Scrobe brillanti, dirette sotto il rostro. Capo sferico, zigrinato. Pronoto più lungo che largo, maggiore ampiezza a metà, leggermente ristretto alle due estremità con punteggiatura poco profonda ma di notevoli dimensioni, intervalli tra i punti, lucidi e di diametro superiore a quello dei punti stessi. Scutello assente. Elitre leggermente arcuate alla base con omeri arrotondati, più larghe del pronoto, strie appenna accennate con punti svaniti e distanziati. Interstrie piane, lisce, con microscopiche setoline visibili solo ai lati ed alla estremità (fig. 1). Zampe sottili, femori appiattiti, tibie sottili con margini interni sinuosi, $3^{\circ}$ articolo dei tarsi bilobo. Sterniti addominali e toracici simili a quelli delle altre specie; sutura tra il $1^{\circ}$ ed il $2^{\circ}$ urosternite obsoleta, non visibile esternamente. Spermateca, ovopositore ed ultimo urosternite: figg. 3-5.

Derivatio nOMinis. Con il nome «nitidipennis» si è voluto sottolineare la più significativa caratteristica morfologica di questa specie.

Note ECOLOICHE. L'esemplare in questione è stato raccolto nella grotta «Aghios Ioannis», quota 110. È il primo reperto di Ruffodytes segnalato per l'ambiente cavernicolo.

Note BIOGEOGRAFICHE. La scoperta di questa nuova specie è di grandissimo interesse non solo perchè amplia grandemente l'areale sinora conosciuto del genere Ruffodytes ma anche perchè ne precisa meglio il significato biogeografico già altrove illustrato (Osella 1976; 1982; Osella-Magnano 1986). Si tratta cioè di paleoendemiti egeici meridionali (fig. 6). È presumibile altresì che il genere Ruffodytes si estenda anche alla penisola anatolica.

\section{Chiave Dicotomica di Ruffodytes Osella, 1973}

Le cinque specie sinora conosciute di Ruffodytes Osella, 1973 possono così essere dicotomicamente riconosciute (vedi anche OSELLA, 1982).

$1^{\circ}$ - Punteggiatura delle elitre fine e superficiale, strie indistinte con punti svaniti e distanziati. Lunghezza $\mathrm{mm} 4,46$. Iraklia (Cicladi) ........... nitidipennis $\mathrm{n}$. $\mathrm{sp}$.

- Punteggiatura delle elitre robusta, strie sempre nettamente evidenti . . . . . .

$2^{\circ}$ - Punteggiatura del pronoto fine e spaziata con traccia di carena mediana sul disco; pronoto più largo delle elitre. Lunghezza mm 6,06. Calabria (Sambiase) ....................................... pacei Osl., 1976

- Punteggiatura del pronoto nettamente più robusta, talora anche variolosa . 


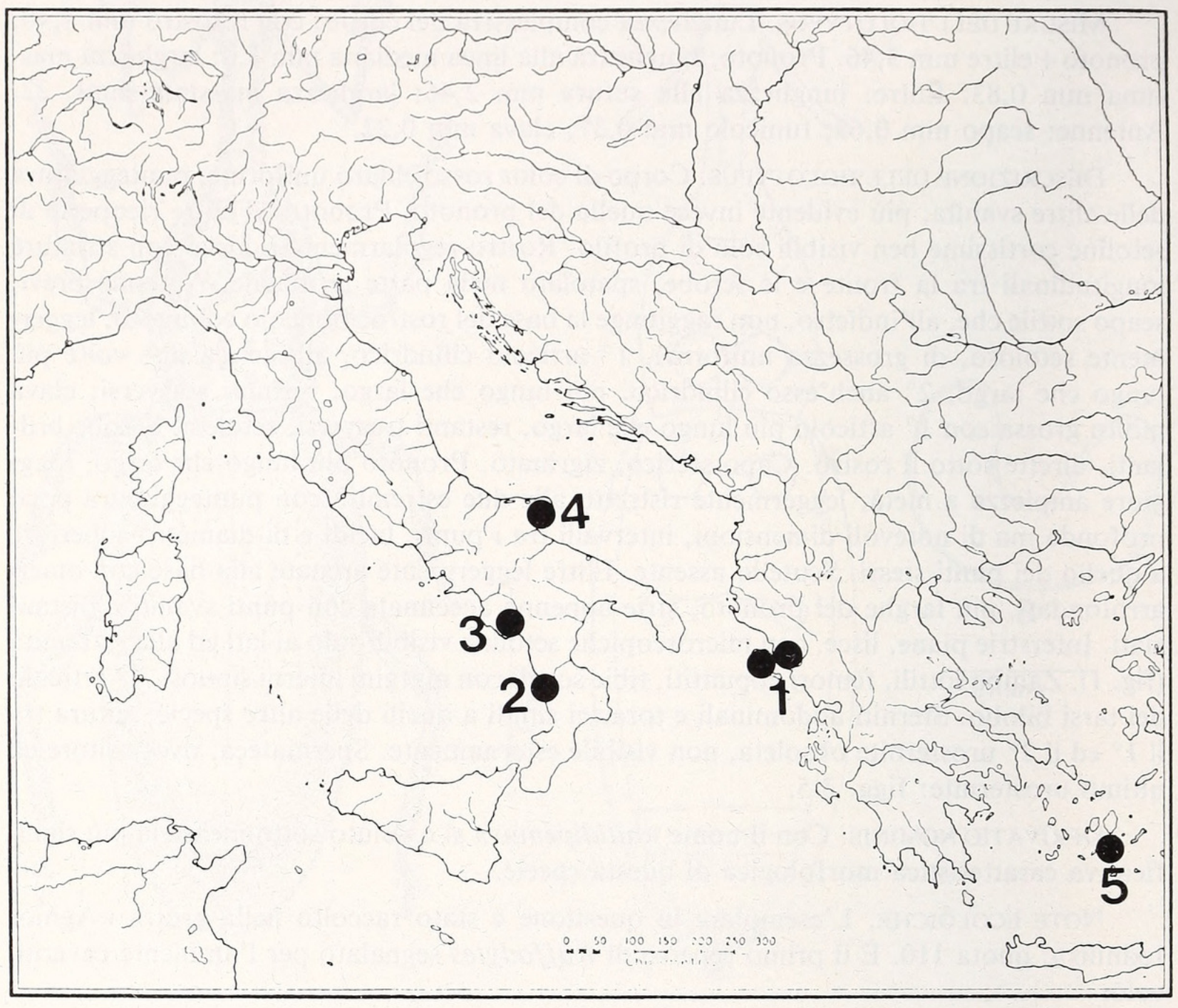

FIG. 6.

Corologia del genere Ruffodytes (Osl.): R. hellenicus (Corfù ed Epiro) (1); R. pacei (Sambiase, Calabria) (2); R. italicus (Acerno, Campania) (3); R. servadeii (Bosco Manatecco, Gargano) (4); R. nitidipennis n. sp. (Iraklia, Cicladi) (5).

$3^{\circ}$ - Punti del pronoto grandi, variolosi, non divisi medialmente da un rilievo; elitre fortemente punteggiate, larghe quanto il pronoto, tibie anteriori inermi, femori mediani con un piccolo dentino. Lunghezza mm 4-5. Corfù ed Epiro ..................................... hellenicus Osl., 1973

- Punti del pronoto più o meno divisi da un rilievo; elitre più strette del pronoto nel punto della loro maggiore ampiezza . . . . . . . . . . . . . . .

$4^{\circ}$ - Dente dei femori mediani e posteriori assai ridotto; edeago largo ad apice leggermente arrotondato. Lunghezza mm 5,6. Campania (Acerno) . . . . . . . . . .

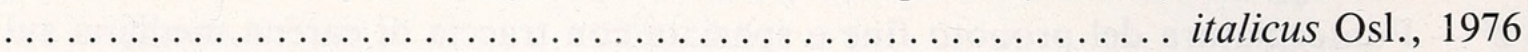

- Dente dei femori mediani e posteriori nettamente più grande ed aguzzo; edeago più stretto e debolmente appuntito. Lunghezza mm 5,01. Gargano (Bosco Manatecco) .............................. servadeii Osl., 1982 
Pseudoalaocybites (Croizatius?) aelleni n. sp. (figg. 7-8)

Loc. tip.: Curaçao (Antilles)

Materiale esaminato. 1 q etichettata «Curaçao, Grot van Hato, 11.II.1985, leg. V. Aellen et P. Strinati»; holotypus: Mus. Ginevra.

Diagnosi. Uno Pseudoalaocybites distinguibile con tutta facilità dalle specie sinora conosciute per la punteggiatura sia del pronoto sia delle elitre finissima e per le zampe molto sottili ed allungate nonchè per lo scutello molto largo (fig. 7).

Misure DELL'HOLOTYPUS. Lunghezza complessiva del corpo: con il rostro mm 3,05; senza rostro $\mathrm{mm} \mathrm{2,38}$. Pronoto: lunghezza alla linea mediana $\mathrm{mm} 0,88$; larghezza mas-

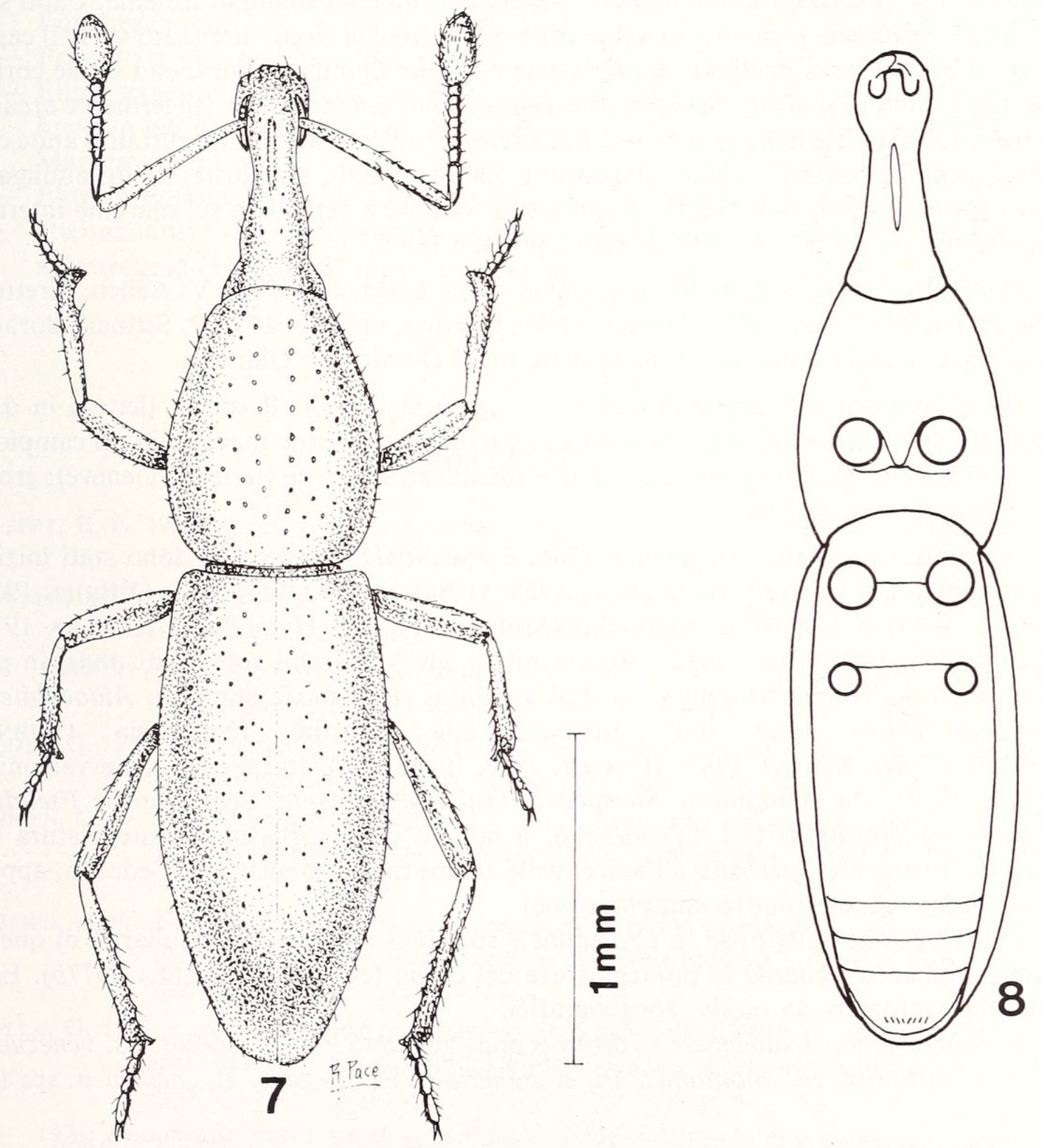

FIGG. 7-8.

Pseudoalaocybites (Croizatius?) aelleni n. sp., Curaçao (Indie Olandesi), Grot van Hato, holotypus ᄋ : habitus (7), schematico dal ventre (8). 


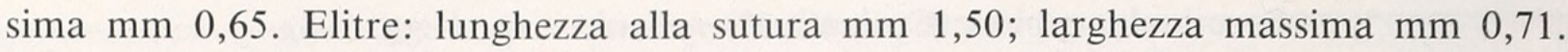

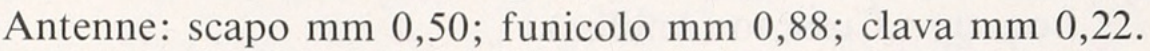

DESCRIZIONE DELL'HOLOTYPUS. Stretto, allungato, di color rosso chiaro, anoftalmo, finemente punteggiato e rivestito di finissime setole. Rostro ad apice spatoliforme, leggermente ricurvo, liscio, con una decina di punti sparsi lungo i margini dorsali e con brevissime setole. Scrobe appena accennate. Strozzatura alla base del rostro evidente sul lato superiore ma svanita ai lati ed inferiormente. Antenne molto snelle, inserite nei $2 / 3$ distali (cioè appena prima della dilatazione spatoliforme); scapo cilindrico, gradatamente ingrossato che raggiunge, all'indietro, il capo; funicolo a $1^{\circ}$ articolo allungato (lungo quanto i tre articoli seguenti sommati insieme), $2^{\circ}$ conico, lungo quanto largo, restanti trasversi di larghezza via via crescente; clava di quattro articoli, moderatamente setolosa, molto grande, lunga quanto gli ultimi quattro articoli del funicolo sommati insieme. Capo sferico, liscio e brillante. pronoto circa due volte più lungo che largo, attenuato verso il capo, piano sul disco, liscio, brillante con finissima e spaziata punteggiatura, con setole cortissime, ben visibili solo ai lati. Scutello triangolare molto grande. Elitre leggermente arcuate alla base, subparallele nella prima metà poi attenuate all'estremità; strie elitrali svanite con punti appena percettibili, saldate alla sutura. Zampe gracili, appiattite, molto allungate, femori piatti e larghi, tibie lineari, leggermente sinuose e dentellate sul margine interno, tarsi stretti, con $3^{\circ}$ articolo non dilatato, pretarsi brevi.

DeriVATIO NOMINIS. Questa interessante specie è dedicata al Dr. V. Aellen, direttore del Museo di Storia Naturale di Ginevra che la raccolse, insieme al Dr. P. Strinati, durante le sue ricerche sulla fauna cavernicola delle Indie Occidentali Olandesi.

Note ecologiche. Secondo quanto mi scrive il Dr. Cl. Besuchet (lettera in data 20.XII.1988) l'unico esemplare rinvenuto di questa specie venne raccolto in un campione di «... sol graveleux mélangé de guano pulvérulent (extraction au Berlese à Genève); grotte sèche; température $27,5^{\circ}$ ».

Note sistematiche. Alaocybites Gilb. e Pseudoalaocybites Osl. sono stati inizialmente attribuiti ai Cossoninae (GILBERT 1955; O'BRIEN \& WIBNER 1982; O'BRIEN 1984; Howden 1983) o alla tribù Anchonini (Molytinae, olim, Hylobiinae) (OSELla 1976; $1977 a ; 1977 b ; 1979 ; 1980 ; 1982)$. Recentemente gli Anchonini sono stati divisi in due tribù; quelli del Nuovo Mondo sono stati attribuiti alla tribù Lymantini. Alaocybites e Pseudoalaocybites sono stati attribuiti alla subtribù Lymantina (WIBNER \& O'BRIEN 1986; KUSHEL 1987; Howden 1988, in stampa). In base alle osservazioni di KUSHEL (1987), che sinonimizza Stewpeckia Osl. con Lymantes Schh., anche Pseudoalaocybites squamirostris Osl. (Venezuela), a motivo della fortissima punteggiatura del corpo, del rostro non spatolato all'apice, delle zampe molto robuste e dell'edeago, appartiene a questo genere (nuova combinazione).

L'attribuzione della n. sp. a Croizatius è solo presunta data la peculiarità di questa specie per quanto riguarda la punteggiatura del corpo (cfr. anche OsELLA 1977b). Essa è tuttavia confortata da motivi zoogeografici.

In conclusione, al subgenere Croizatius appartengono le segg. specie: Ps. venezuelanus, Ps. latithorax, Ps. montanus, Ps. margheritae, Ps. elegans, Ps. aelleni n. sp. (?).

\section{Chiave dicotomica di Pseudoalaocybites SubG. Croizatius OSElla, 1980}

$1^{\circ}$ - Elitre e pronoto a punteggiatura finissima con zampe gracili ed allungate, scutello assente. Lunghezza $\mathrm{mm} 3,05$. Curaçao .............. aelleni n. sp.

- Elitre e pronoto a punteggiatura robusta, zampe più corte scutello presente 2 
$2^{\circ}$ - Pronoto più stretto, alla base, delle elitre; pronoto ed elitre con punteggiatura spaziata, poco profonda; edeago allungato con apice subtroncato. Lunghezza mm 2,88. Maracay (Venezuela) .....................elegans Osl., 1987

- Pronoto largo quanto le elitre; pronoto ed elitre con punteggiatura più fitta

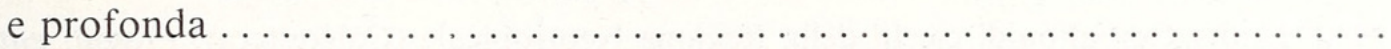

$3^{\circ}$ - Pronoto molto grande e robusto, largo, nella parte mediana, quanto le elitre nel punto della maggiore ampiezza. Elitre fortemente striate e punteggiate. Edeago allungato con apice arrotondato. Lunghezza $\mathrm{mm} 4,06$. Quindio

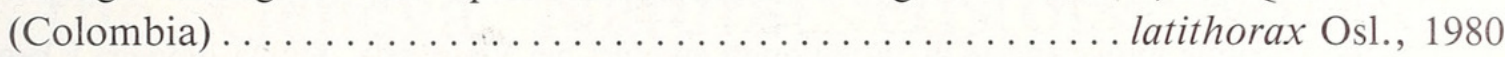

- Pronoto meno robusto, meno largo, nella zona mediana, delle elitre nel punto

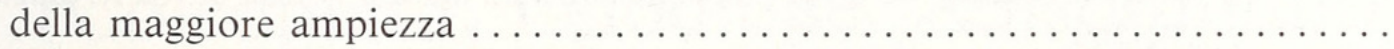

$4^{\circ}$ - Pronoto finemente e fittamente punteggiate con punti di media grandezza. Lunghezza mm 3,32. Calarca (Colombia) ............. montanus Osl., 1980

- Pronoto a punteggiatura più grande e robusta; rostro ad apice ampliato...

$5^{\circ}$ - Elitre leggermente arcuate alla base, strie con robusta punteggiatura. Edeago breve, a lati paralleli con apice largamente arrotondato. Lunghezza mm 2,85.

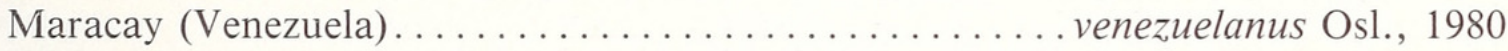

- Elitre a doppia sinuosità alla base, strie con punti meno robusti che vanno attenuandosi verso l'estremità. Edeago più largo, leggermente stretto all'estremità. Lunghezza mm 5,0. Aragua (Venezuela) .... margheritae Osl., 1980

\section{BIBLIOGRAFIA}

Gilbert, E. E. 1955. A new genus and species of blind weevil from Florida. Pan-Pacif. Ent., 31: 193-195.

Howden, A. T. 1988. Two new species of Decuanellus Osella from Virgin Islands (Coleoptera: Curculionidae: Cossoninae). Coleopts. Bull., 37: 81-89.

- 1989. Review of the Caribbean subtribe Caecossonina with description of new taxa (Coleoptera: Curculionidae) (in stampa zu Quaest. entom.).

Kushel, G. 1987. The subfamily Molytinae (Coleoptera: Curculionidae): General notes and description of new taxa from New Zealand and Chile. Neue. Z. Ent., 9: 11-29.

O'Brien, C. W. 1984. Paralicus minyops O'Brien, new genus and species of Cossoninae from Florida and Bahama Islands (Coleoptera: Curculionidae). SWest. Entomol., 9 (3): 346-349.

O'Brien, C. W. \& G. J. Wibner. 1982. Annoted checklist of the weevils (Curculionidae sensu lato) of North America, Central America and West Indies (Coleoptera: Curculionidae). Mem. Am. ent. Inst., 34: 1-382.

Osella, G. 1973. Una nuova specie di cossonino cieco dell'isola di Cuba (Coleoptera, Curculionidae). Résultats des expéditions biospéologiques cubano-roumaines à Cuba. Acad. R. S. R. Bucuresti, 1: 373-377.

- 1976. Decuanellus viti n. sp. di curculionide endogeo dell'isola di Guadalupa (Coleoptera). Revue suisse Zool., 83: 673-677.

- 1977a. Pseudocaecocossonus zayasi n. gen. n. sp. di curculionide endogeo di Cuba (Coleoptera). Résultats des expéditions biospéologiques cubano-roumanes à Cuba. Acad. R. S. R. Bucuresti, 2: 390-395. 
Osella, G. 1977b. Nuove specie di curculionidi endogei dell'isola di Cuba (Coleoptera). Résultats des expéditions biospéologiques cubano-roumaines à Cuba. Acad. R. S. R. Bucuresti, 2: 397-402.

- 1979. Soil Curculionidae (Coleoptera). Boll. Zool., 46: 299-318.

- 1980. Nuovi Curculionidi ciechi della Regione Neotropicale (Coleoptera). Fragm. ent., 15: $365-422$.

- 1982. Ruffodytes servadeii n. sp. di Erirrhininae del Gargano. Mem. Soc. ent. ital. Genova, 60: 273-277.

- 1987. Cinque nuove specie di curculionidi endogei del Venezuela. Fauna hipogea y hemiedafica de Venezuela y de otros paises de America del Sur. Acad. R. S. R. Bucuresti, 1: 201-209.

Osella, G. \& L. Magnano. 1984 (1986). I coleotteri attelabidi e curculionidi a diffusione transadriatica. Biogeographia. Lav. Soc. ital. Biogeogr., n. s., 10: 701-792.

WibNer, G. J. \& C. W. O’Brien. 1986. Annoted checklist of the weevils (Curculionidae sensu lato) of South America (Coleoptera: Curculionidae). Mem. Am. ent. Inst., 39: 1-563. 


\section{$2 \mathrm{BHL}$ Biodiversity Heritage Library}

1989. "Due nuovi curculionidi endogei (Coleoptera) (34. Contributo alla conoscenza della curculionidofauna endogea)." Revue suisse de zoologie 96, 451-458. https://doi.org/10.5962/bhl.part.82043.

View This Item Online: https://www.biodiversitylibrary.org/item/128795

DOI: https://doi.org/10.5962/bhl.part.82043

Permalink: https://www.biodiversitylibrary.org/partpdf/82043

\section{Holding Institution}

Smithsonian Libraries

\section{Sponsored by}

Biodiversity Heritage Library

\section{Copyright \& Reuse}

Copyright Status: In Copyright. Digitized with the permission of the rights holder.

Rights Holder: Muséum d'histoire naturelle - Ville de Genève License: http://creativecommons.org/licenses/by-nc-sa/3.0/ Rights: https://www.biodiversitylibrary.org/permissions/

This document was created from content at the Biodiversity Heritage Library, the world's largest open access digital library for biodiversity literature and archives. Visit BHL at https://www.biodiversitylibrary.org. 